Карин ПУННИНГ, Т. СЫМЕР, А. ТРУММЕЛ

\title{
ИЗУЧЕНИЕ ОПТИМАЛЬНОГО РАБОЧЕГО РЕЖИМА ФЛЮИД- НОГО ХРОМАТОГРАФА НА ПРИМЕРЕ АНАЛИЗА ХЛОРИСТОГО БЕНЗИЛА
}

\author{
(Представил О. Киррет)
}

В последние годы существенно расширились исследования в области флюидной хроматографии. Применение элюентов в критическом или сверхкритическом состояниях открыло новые перспективы в развитии хроматографии. Однако флюидная хроматография предъявляет жесткие требования к инструментальным характеристикам прибора. Прибор должен обеспечить регулировку давления, температуры и скорости потока элюента с высокой точностью. В данной работе описан наш опыт изучения оптимального рабочего режима флюидного хроматографа на примере анализа хлористого бензила.

\section{Экспериментальная часть}

Флюидный хроматограф был собран на базе жидкостного хроматографа AVK-31, разработанного в СКБ АН ЭССР [']. Двухплунжерный насос обеспечивал подачу элюента от 0,1 до $9,9 \mathrm{~cm}^{3} /$ мин. В качестве элюента использовали $\mathrm{CO}_{2}$. Его критическое давление 7,38 МПа и критическая температура $31,06{ }^{\circ} \mathrm{C}\left[{ }^{2}\right]$. Для стабилизации режима подачи жидкого $\mathrm{CO}_{2}$ цилиндры насоса покрывали специальными рубашками и пропускали через них жидкий азот для поддержания температуры в цилиндрах от -3 до $-10^{\circ} \mathrm{C}$. Температуру в них регистрировали встроенной термопарой хромель-копель.

В качестве детектора использовали УФ-детектор жидкостного микроколоночного хроматографа «Милихром» (разработанного в Новосибирском институте органической химии СО АН СССР) с объемом ячейки 0,8 мкл, модифицированной нами для работы при высоких давлениях (до 15 МПа). Измерения проводили на длине волны 220 нм.

Регулятор высокого давления расположен в системе за детектором. Давление элюента измеряли с помощью манометров высокого давления перед петлевым дозатором (на входе) и после детектора (на выходе). Расход $\mathrm{CO}_{2}$ определяли ротаметром и измерителем с бюреткой после декомпрессии $\mathrm{CO}_{2}$ и пересчитывали для нормальных условий $\left(0^{\circ} \mathrm{C}\right.$ и 0,1 МПа).

Разделяющие колонки из нержавеющей стали, размерами $150 \times 4$ мм, были заполнены Силасорбом $\mathrm{C}_{18}$ или Силасорбом 600 фирмы «Lachema» (ЧССР). Размер частиц 5 мкм. Термостат колонок обеспечивал нагрев до $200^{\circ} \mathrm{C}$, стабильность поддержания температуры $\pm 0,1^{\circ} \mathrm{C}$. Перепад давления в колонке не превышал 1,0 МПа.

Для очистки $\mathrm{CO}_{2}$ (I copт, ГОСТ 8050-85) служила вмонтированная перед насосом колонка $(500 \times 8$ мм), заполненная молекулярными ситами $\mathrm{NaX}$. Колонка $(150 \times 20$ мм) с силикагелем 40/100 находилась в системе между насосом и петлевым дозатором. Раствор хлористого бен- 
зила в гексане объемом 5 мкл вводили в систему с помощью петлевого дозатора. Концентрация хлористого бензила составляла 0,1 и $0,02 \%$ об.

Для выявления технических параметров собранного флюидного хроматографа были поставлены следующие эксперименты:

1. Изучение влияния степени очистки $\mathrm{CO}_{2}$ на время удерживания хлористого бензила в зависимости от расхода $\mathrm{CO}_{2}$ при заданных условиях эксперимента.

2. Определение предела обнаружения хлористого бензила в завйсимости от температуры и давления на выходе при разных расходах $\mathrm{CO}_{2}$. Разделяющая колонка была заполнена Силасорбом 600.

3. Установление эффективности разделяющей колонки, заполненной Силасорбом 600 , в зависимости от изменения температуры и давления на выходе при разных линейных скоростях потока элюента.

\section{Результаты и их обсуждение}

Из рис. 1 видно, что время удерживания хлористого бензила существенно зависит от степени очистки $\mathrm{CO}_{2}$ при заданных температуре, давлении на выходе и расходе $\mathrm{CO}_{2}$. C применением в качестве носителя Силасорба 600 очистка $\mathrm{CO}_{2}$ увеличивает время удерживания хлористого бензила, что говорит о возможном содержании в $\mathrm{CO}_{2}$ полярных примесей, которые уже в низких концентрациях могут сильно влиять на селективность колонки и воспроизводимость времени удерживания [ ${ }^{3}$. Поэтому во всех остальных экспериментах $\mathrm{CO}_{2}$ очищали, пропуская его через колонки, заполненные молекулярными ситами $\mathrm{NaX}$ и силикагелем.

Изучение оптимального рабочего режима хроматографа для количественного химического анализа требует выяснения факторов, влияющих на предел обнаружения $Q_{0}$. Судя по рис. $2, Q_{0}$ хлористого бензила

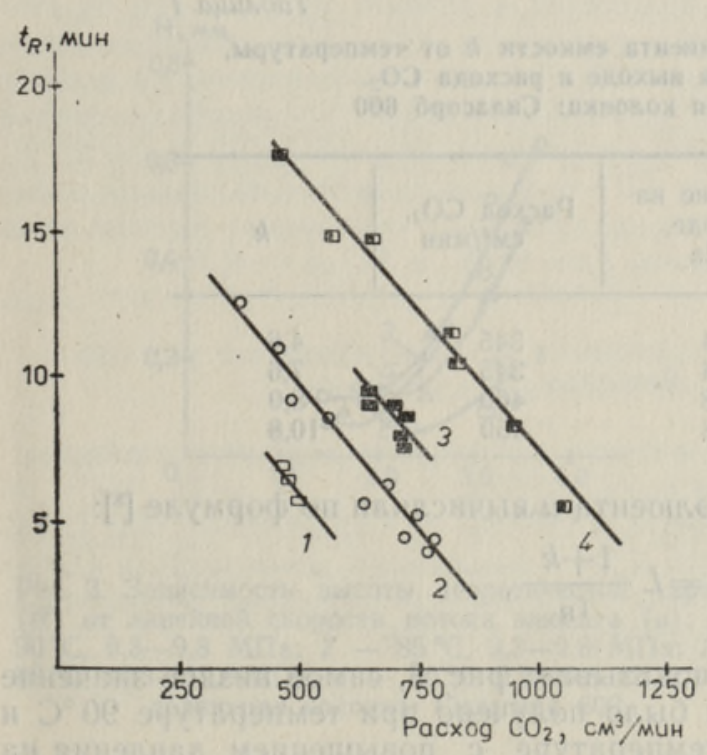

Рис. 1. Зависимость времени удерживания хлористого бензила $\left(t_{R}\right)$ от расхода неочнщенного $\mathrm{CO}_{2}(1,2)$ и пропущенного сначала через колонку с молекулярными ситами $\mathrm{NaX}$ (3), а потом через колонку с силикагелем (4). Разделяющая колонка: 1 - Силасорб $\mathrm{C}_{18} ; 2-4$ - Снласорб 600 . Температура $80^{\circ} \mathrm{C}$, давление на выходе $9,6 \mathrm{MПа.}$

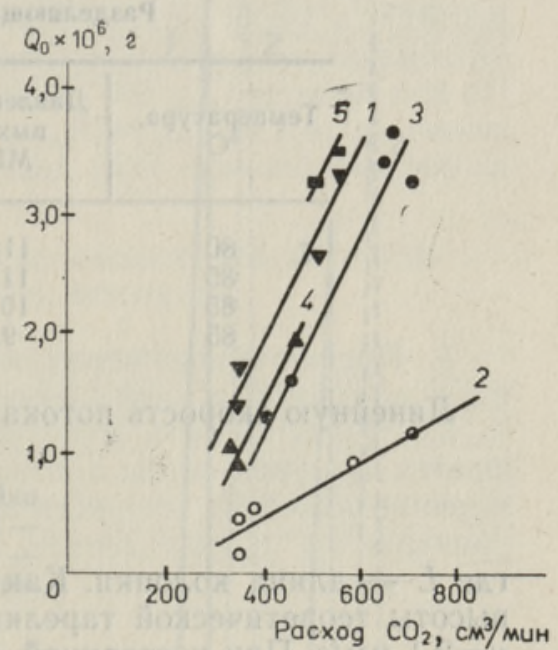

Рис. 2. Предел обнаружения хлористого бензнла $\left(Q_{0}\right)$ в зависимости от расхода $\mathrm{CO}_{2}$ при температурах $70(1), 80(2,3), 85(4)$ и $90^{\circ} \mathrm{C}(5)$ и давлениях на выходе $8,8-9,0$ ( 1 , 3-5) и 11,3-11,8 МПа (2). Разделяющая колонка: Силасорб 600. 
уменьшается с повышением давления на выходе и уменьшением расхода $\mathrm{CO}_{2}$. Это обусловлено падением уровня флуктуационных шумов детектора при низких расходах $\mathrm{CO}_{2}$. В то же время при повышении давления на выходе наблюдается сжатие пика [ $\left.{ }^{4}\right]$, что ведет к увеличению его высоты. Например, при вводе в колонку пробы, содержащей $1,1 \cdot 10^{-4}$ г хлористого бензила (температура $80^{\circ} \mathrm{C}$ и расход $\mathrm{CO}_{2} 310 \mathrm{~cm}^{3} / \mathrm{Mин}$ ), ширина пика на половине высоты $W_{h}$ при 9,1 МПа составляет 66 с, при 11,4 МПа - 40 с. Высота пика при этом увеличивается в 2,3 раза.

По данным [5], растворяющая способность $\mathrm{CO}_{2}$ возрастает с ростом ero плотности, причем при постоянной плотности она мало зависит от температуры. Известно также, что при давлениях, намного выше критического, плотность $\mathrm{CO}_{2}$ резко уменьшается с повышением температуры $\left.{ }^{6}\right]$. При постоянной температуре, выше критической, повышение давления вызывает увеличение плотности $\mathrm{CO}_{2}\left[{ }^{7}\right]$, в результате чего растворимость хлористого бензила в элюенте возрастает и время его удерживания уменьшается. С этим согласуются и данные наших экспериментов, проведенных при $80^{\circ} \mathrm{C}$ и расходе $\mathrm{CO}_{2} 620 \mathrm{~cm}^{3} /$ мин. Так как плотность $\mathrm{CO}_{2}$ и, следовательно, его растворяющая способность при 11,7 МПа выше, чем при 10,6 МПа, то время удерживания хлористого бензила составляло, соответственно, 4,2 и 21,0 мин.

Коэффициент емкости $k$ рассчитывали по формуле [8]:

$$
k=\left(t_{R}-t_{0}\right) / t_{0},
$$

где $t_{0}$ и $t_{R}$ - времена удерживания гексана и хлористого бензила соответственно. Как показывают данные табл. 1, при постоянном давлении на выходе и постоянном расходе $\mathrm{CO}_{2} k$ увеличивается с повышением температуры. Из-за уменьшения плотности $\mathrm{CO}_{2}\left[{ }^{2}\right]$ растворимость хлористого бензила в элюенте при $85^{\circ} \mathrm{C}$ меньше, чем при $80^{\circ} \mathrm{C}$, а время его удерживания, соответственно, при $80^{\circ} \mathrm{C}$ меньше, чем при $85^{\circ} \mathrm{C}$.

таблица 1

Зависимость коэффициента емкости $k$ от температуры, давления на выходе и расхода $\mathrm{CO}_{2}$.

Разделяющая колонка: Силасорб 600

\begin{tabular}{c|c|c|}
\hline Tемпература, & $\begin{array}{c}\text { Давление на } \\
\text { выходе, } \\
\text { МПа }\end{array}$ & $\begin{array}{c}\text { Расход } \mathrm{CO}_{2}, \\
\mathrm{~cm}^{3} / \text { мин }\end{array}$
\end{tabular} \mid $k$

$\begin{array}{rrrr}80 & 11,4 & 345 & 4,6 \\ 85 & 11,4 & 345 & 7,6 \\ 85 & 10,8 & 460 & 8,0 \\ 85 & 9,8 & 460 & 10,8\end{array}$

Линейную скорость потока элюента $u$ вычисляли по формуле [8]:

$$
u=L \frac{1+k}{t_{R}},
$$

где $L-$ длина колонки. Қак показывает рис. 3 , самое низкое значение высоты теоретической тарелки было получено при температуре $90^{\circ} \mathrm{C}$ и $u=2,1 \mathrm{~mm} / \mathrm{c}$. При постоянной температуре с повышением давления на выходе уменьшается оптимальная линейная скорость потока элюента, соответствуя минимальному значению высоты теоретической тарелки $H$. Известно, что динамическая вязкость $\mathrm{CO}_{2}$ уменьшается с понижением давления при постоянной температуре []. Это вызывает увеличение скорости диффузии хлористого бензила в $\mathrm{CO}_{2}$. Для предотвращения возможного размывания пика из-за увеличения скорости диффузии [10] 
необходимо повысить линейнную скорость потока элюента. На размывание пика указывает и то, что его ширина на половине высоты увеличивается от 37 до 59 с с уменьшением давления на выходе от 11,3 до 10,3 МПа при $85^{\circ} \mathrm{C}$ и $u=1,8 \mathrm{~mm} / \mathrm{c}$. По названным причинам в проведенном нами эксперименте при $85^{\circ} \mathrm{C}$ оптимальная скорость потока элюента при 11,3 МПа составляет 1,75 мм/с, а при 10,3 МПа $-2,25 \mathrm{mм} / \mathrm{c}$.

При постоянной линейной скорости потока элюента и при уменьшении температуры плотность $\mathrm{CO}_{2}$ увеличивается [1']. В нашем эксперименте при $u=2,25 \mathrm{~mm} / \mathrm{c}$ плотность $\mathrm{CO}_{2}$ увеличивается и, соответственно, коэффициент диффузии хлористого бензила уменьшается при понижении температуры от 90 до $85^{\circ} \mathrm{C}$. Но известно, что высокие значения коэффициента диффузии определяемого вещества обеспечивают большую скорость массообмена со стационарной фазой и тем самым гарантируют хорошие разделяющие характеристики []. В результате этого эффективность колонки при $90^{\circ} \mathrm{C}$ должна быть выше, чем при $85^{\circ} \mathrm{C}$, что и подтверждается данными, полученными нами (см. табл. 2). Однако надо отметить, что при повышении температуры эффективность колонки может улучшаться и под влиянием воды, содержащейся в $\mathrm{CO}_{2}\left[{ }^{12,13}\right]$. Известно, что растворимость воды в $\mathrm{CO}_{2}$ увеличивается с повышением температуры, в результате чего увеличивается число свободных $-\mathrm{Si}-\mathrm{OH}$ -групп. Так как энергия адсорбции анализируемого вещества на этих группах выше, чем на $-\mathrm{Si}-\mathrm{OH} \cdot n \mathrm{H}_{2} \mathrm{O}$-группах, увеличивается время удерживания анализируемого вещества. На возможное содержание воды в $\mathrm{CO}_{2}$ указывают и данные, приведенные на рис. 1.

На типичных хроматограммах смеси хлористого бензила в гексане при разных давлениях на выходе и расходах $\mathrm{CO}_{2}$, но при постоянной температуре (рис. 4) пик гексана заметен только при высоких давле-

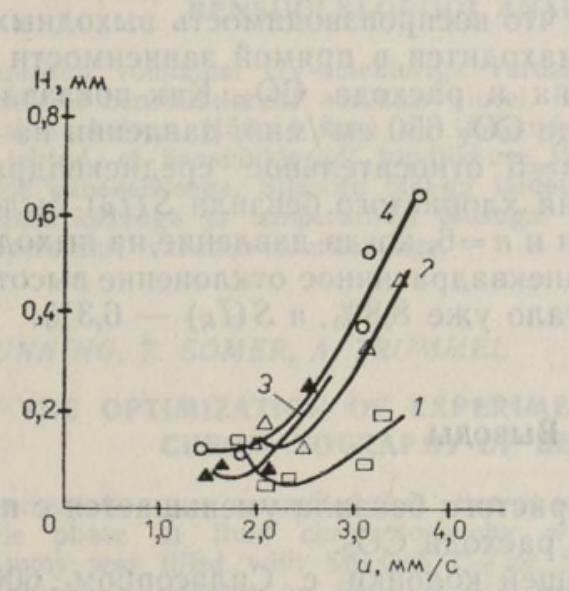

Рнс. 3. Зависимость высоты теоретической тарелки $(H)$ от линейной скорости потока элюента $(u): 1-$ $90{ }^{\circ} \mathrm{C}, 9,3-9,8 \mathrm{M \Pi а} ; 2-85^{\circ} \mathrm{C}, 9,3-9,8 \mathrm{MПа} ; 3-$ $85^{\circ} \mathrm{C}, 10,3-10,8$ МПа; $4-80^{\circ} \mathrm{C}, 9,3-9,8$ МПа. Разделяющая колонка: Силасорб 600.

Рис. 4. Хроматограмма раствора хлористого бензила в гексане при разных расходах $\mathrm{CO}_{2}$ и давлениях на выходе: $1-370 \mathrm{~cm}^{3} /$ мин, $11,4 \mathrm{MПа} ; 2-355 \mathrm{~cm}^{3} / \mathrm{Mин,}$ 12,0 МПа; 3 - $580 \mathrm{~cm}^{3} /$ мин, 10,8 МПа; 4 $580 \mathrm{~cm}^{3} /$ мнн, 12,0 МПа. Разделяющая колонка: Снлаcop6 600. Температура: $80^{\circ} \mathrm{C}$. Проба объемом 5 мкл: $1,1 \times 10^{-4}$ г хлористого бензила в гексане.

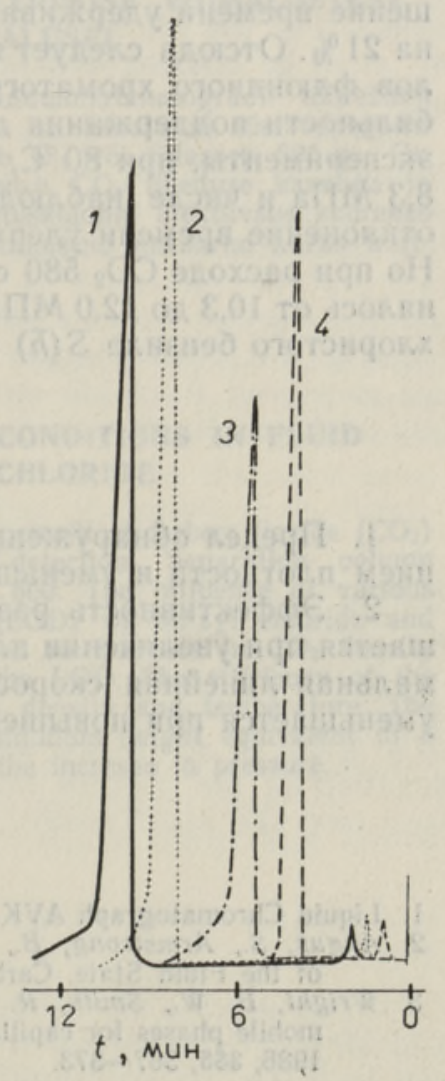


Зависимость числа теоретических тарелок $N$ от линейной скорости потока элюента $u$ при разных температурах и давлениях на выходе.

Разделяющая колонка: Силасорб 600

\begin{tabular}{c|c|c|c}
\hline Температура, & $\begin{array}{c}\text { Давлениена } \\
\text { выходе, } \\
\text { MПа }\end{array}$ & $\begin{array}{c}u, \\
\text { мм/c }\end{array}$ & $\begin{array}{c}N, \\
\text { TT/M }\end{array}$ \\
\hline \multirow{2}{*}{80} & 11,6 & 3,7 & 1553 \\
& 11,6 & 2,5 & 2128 \\
& 11,7 & 3,1 & 3077 \\
85 & 11,7 & 1,4 & 8130 \\
& 12,1 & 1,8 & 9090 \\
& 10,1 & 2,0 & 8064 \\
& 10,6 & 2,5 & 8000 \\
90 & 10,9 & 2,1 & 6024 \\
& 11,1 & 2,5 & 4065 \\
& 11,6 & 1,5 & 17857 \\
& 12,3 & 1,6 & 12195 \\
& 8,1 & 3,1 & 11494 \\
& 8,7 & 2,3 & 17544 \\
& 8,9 & 2,1 & 21739 \\
& 9,2 & 1,8 & 7407
\end{tabular}

ниях на выходе. Повышение давления на выходе на $11 \%$ вызывает, при постоянном расходе $\mathrm{CO}_{2}$, уменьшение времени удерживания хлористого бензила на $34 \%$ и увеличение высоты пика на $32 \%$. При постоянном давлении на выходе увеличение расхода $\mathrm{CO}_{2}$ на $39 \%$ обуславливает уменьшение времени удерживания хлористого бензила на $55 \%$ и высоты пика на $21 \%$. Отсюда следует вывод, что воспроизводимость выходных сигналов флюидного хроматографа находится в прямой зависимости от стабильности поддержания давления и расхода $\mathrm{CO}_{2}$. Как показали наши эксперименты, при $80^{\circ} \mathrm{C}$, расходе $\mathrm{CO}_{2} 650 \mathrm{~cm}^{3} /$ мин, давлении на выходе 8,3 МПа и числе наблюдений $n=5$ относительное среднеквадратичное отклонение времени удерживания хлористого бензила $S\left(\bar{t}_{R}\right)$ было $2,1 \%$. Но при расходе $\mathrm{CO}_{2} 580 \mathrm{~cm}^{3} /$ мин и $n=6$, когда давление на выходе изменялось от 10,3 до 12,0 МПа, среднеквадратичное отклонение высоты пика хлористого бензила $S(\bar{h})$ достигало уже $8,8 \%$, а $S\left(\bar{t}_{R}\right)-6,3 \%$.

\section{Выводы}

1. Предел обнаружения хлористого бензила уменьшается с повышением плотности и уменьшением расхода $\mathrm{CO}_{2}$.

2. Эффективность разделяющей колонки с Силасорбом 600 улучшается при увеличении плотности $\mathrm{CO}_{2}$ и повышении температуры. Оптимальная линейная скорость потока элюента при заданной температуре уменьшается при повышении давления на выходе.

\section{ЛИТЕРАТ У РА}

1. Liquid Chromatograph AVK-31. Tallinn, 1981.

2. Angus, S., Armstrong, B., de Reuck, $K$. M. International Thermodynamic Tables of the Fluid State. Carbon Dioxide, Oxford, New York et al., 1976, 7.

3. Wright, $B . W$., Smith, $R$. D. Investigation of polar modifiers in carbon dioxide mobile phases for capillary supercritical fluid chromatography. - J. Chromatogr., 1986, 355, 367-373. 
4. Wilsch, A., Schneider, G. M. Retention and resolution in density-programmed supercritical fluid chromatography. I. Theory and selected results. - J. Chromatogr., $1986,357,239-252$.

5. Schoenmakers, P. J. Thermodynamic model for supercritical fluid chromatography. J. Chromatogr., 1984, 315, 1-18.

6. Blilie, A. L., Greibrokk, T. Gradient programming and combined gradient-pressure programming in supercritical fluid chromatography. - J. Chromatogr., 1985, $349,317-322$.

7. Gere, D. R., Board, R., McManigill, D. Supercritical fluid chromatography with small particle diameter packed columns. - Anal. Chem., 1982, 54, 736-740.

8. Yost, R. W., Ettre, L. S., Conlon, R. D. Practical Liquid Chromatography. An Introduction. New York, 1980, 47.

9. Lauer, H. H., McManigill, D., Caude, M., Rosset, R. H., Tambute, A. G. Mobilephase transport properties of liquefied gases in near-critical and supercritical fluid chromatography. - Anal. Chem., 1983, 55, 1370-1375.

10. Novotny, M., Springston, S. R. Fundamentals of column performance in supercritical fluid chromatography. - J. Chromatogr., 1983, 279, 417-422.

11. Fields, S. M., Lee, M. L. Effects of density and temperature on efficiency in capillary supercritical fluid chromatography. - J. Chromatogr., 1985, 349, 305-316.

12. Mourier, P., Sassiat, P., Caude, M., Rosset, R. Retention and selectivity in carbon dioxide supercritical fluid chromatography with various stationary phases. J. Chromatogr., 1986, 353, 61-75.

13. Schwartz, H. E., Brownlee, R. G. Hydrocarbon group analysis of gasolines with microbore supercritical fluid chromatography and flame ionisation detection. J. Chromatogr., 1986, 353, 77-93.

Специальное конструкторское бюро

Академии наук Эстонской ССР

Поступила в редакцию 18/VI 1987

Karin PUNNING, T. SOMER, A. TRUMMEL

\section{FLUIDUMIKROMATOGRAAFI TOOOREZIIMI OPTIMEERIMISE VOIMALUSTEST BENSUOLKLORIIDI ANALUUSI ALUSEL}

On uuritud võimalusi UV-detektoriga varustatud fluidumikromatograafi töörežiimi optimeerimiseks bensüülkloriidi analüüsi alusel. Eluendina on kasutatud süsihappegaasi $\left(\mathrm{CO}_{2}\right)$. Lahutuskolonn $(150 \times 4 \mathrm{~mm})$ oli täidetud Silasorb $\mathrm{C}_{18}$ vôi Silasorb 600-ga. On kindlaks tehtud, et bensüülkloriidi tundlikkuse lävi väheneb $\mathrm{CO}_{2}$ tiheduse kasvuga ja mahtkiiruse vähenemisega. Silasorb 600-ga täidetud lahutuskolonni efektiivsus suureneb $\mathrm{CO}_{2}$ tiheduse kasvuga ja temperatuuri tôusuga. $\mathrm{CO}_{2}$ optimaalne lineaarne kiirus uuritaval temperatuuril väheneb rõhu tõusuga.

\section{Karin PUNNING, T. SOMER, A. TRUMMEL}

\section{ON THE OPTIMIZATION OF EXPERIMENTAL CONDITIONS IN FLUID CHROMATOGRAPHY OF BENZYL CHLORIDE}

Instrumentation was assembled that allowed to use supercritical carbon dioxide $\left(\mathrm{CO}_{2}\right)$ as mobile phase in fluid chromatography with UV-detection. Separation column $(150 \times 4 \mathrm{~mm})$ was filled with Silasorb $\mathrm{C}_{18}$ or Silasorb 600 . The influence of various chromatographic conditions on the limit of detection (LOD) of benzyl chloride and column performance was investigated. It was found that an increase in $\mathrm{CO}_{2}$ density and a decrease in flow-rate resulted in a decrease of the LOD. The efficiency of the Silasorb 600 column increases with the increase in $\mathrm{CO}_{2}$ density and temperature. The optimum linear velocity of $\mathrm{CO}_{2}$, corresponding to the minimum height equivalent to a theoretical plate, at constant temperature decreases with the increase in pressure. 